\title{
Late onset multiple sclerosis - multiparametric MRI characteristics
}

\author{
Łukasz Jasek ${ }^{1}$, Janusz Śmigielski ${ }^{2}$, Małgorzata Siger ${ }^{3}$ \\ ${ }^{1}$ Department of Neurology, Stroke and Neurorehabilitation, Medical University of Lodz, Lodz, Poland \\ ${ }^{2}$ State Higher Vocational School, Konin, Poland \\ ${ }^{3}$ Department of Neurology, Medical University of Lodz, Lodz, Poland
}

\begin{abstract}
Introduction. Multiple sclerosis (MS) is a demyelinating disease of the central nervous system (CNS) with heterogenic character. Typical age of onset is between 20 and 35 years. Clinically definite multiple sclerosis (CDMS) can occur also in patients older than 50 years. This type of MS is called Late Onset Multiple Sclerosis (LOMS). Until now, the differences in clinical course, type of first symptoms, and prognosis of LOMS have not been well established. Also the MRI characteristics of patients with LOMS have not been determined. Neither conventional nor nonconventional MRI features are known to be typical for LOMS.

Clinical rationale for the study. To investigate the MRI characteristics of LOMS patients based on conventional and non-conventional techniques.
\end{abstract}

Materials and methods. Twenty patients with LOMS were included in the study and 17 patients with typical onset of MS (TOMS) served as a comparative group. The two groups were matched in terms of disease duration and EDSS score. Conventional (T1- and T2-weighted images) and non-conventional (magnetization transfer images, proton magnetic resonance spectroscopy) MRI techniques were performed in all participants. Parameters from both techniques were compared between LOMS and TOMS groups.

Results. Patients with late onset of MS had lower Brain Parenchyma Fraction (BPF) $(p<0.001)$ and Grey Matter Fraction (GMF) values $(p=0.008)$ than the TOMS group. There was no statistical differences in White Matter Fraction (WMF) values between the groups $(p=0.572)$. Patients with LOMS and TOMS statistically differed in the peak height $(p=0.018)$, peak location $(p<0.001)$, and MTR mean value $(p<0.001)$. Patients with LOMS manifested lower concentrations of NAA+NAAG and NAA+NAAG/Cr than patients with TOMS $(p=0.009$ and $p<0.001$ respectively). No statistical difference was found between the groups in terms of mean $\mathrm{mln}(\mathrm{p}=0.346)$ and mean GPC+PCh $(p=0.563)$. We did not find a statistical difference in T1- and T2- lesion load $(p=0.1$, $\mathrm{p}=0.3$ respectively) although $\mathrm{T} 1 / \mathrm{T} 2$ lesion ratio was higher in the LOMS group.

Conclusion and clinical implications. MRI parameters in patients with LOMS differed significantly from those obtained from the TOMS group. Our results, which indicate that in LOMS patients brain tissue damage is more advanced than in TOMS patients, may contribute to a better understanding of the heterogeneity of MS.

Key words: Late onset multiple sclerosis, multiple sclerosis, magnetic resonance imaging, brain atrophy, proton magnetic resonance spectroscopy, magnetization transfer ratio

(Neurol Neurochir Pol 2020; 54 (3): 265-271)

\section{Introduction}

In the typical course of multiple sclerosis (MS), the first symptoms occur between the ages of 20 and 35 years. However, according to epidemiological findings, in $4.6-9.6 \%$ of MS cases the first symptoms are observed in patients aged over $50[1,2,3]$. This form of the disease is referred to as Late Onset Multiple Sclerosis (LOMS) [4-8]. Very recently published results from Denmark have shown that the incidence of LOMS has increased since the 1950s, particularly in women [9]. The

Corresponding author: Łukasz Jasek, Department of Neurology, Stroke and Neurorehabilitation, Medical University of Lodz, Kopcińskiego 20 Str., 90-153 Lodz, Poland, e-mail: lukaszjjasek@gmail.com 
population of these MS patients clinically differs from the population of patients who are affected by MS at the typical age (Typical Age of Onset Multiple Sclerosis, TOMS). In LOMS patients, the primary progressive form of the disease (PPMS) is more common than in TOMS (50-80\% in LOMS vs $10-20 \%$ in TOMS) [5-7, 10-13]. The first symptoms of MS in LOMS and TOMS are also different. In LOMS, cerebellar and sensory symptoms have been reported to be much more common than in TOMS [4,5]. Gait disturbances with spastic paraparesis are the most common motor symptoms of LOMS [6]. The prognosis in the LOMS form of the disease is less favourable than for patients with the TOMS form [5, 6, 14]. LOMS patients seem to progress more rapidly than TOMS, especially with the primary progressive course of disease $[13,15,16]$. LOMS has also been associated with a severe disease course and has been found to be a strong predictor of conversion from RR to secondary progressive MS [14]. Additionally, there has been shown to be no impact of interferon beta treatment on disability progression in RR- LOMS patients [13]. Although magnetic resonance imaging is an established and very important paraclinical tool in the diagnosis of MS, there is very limited data concerning MRI in LOMS patients. In one study [11], the sensitivity and specificity of radiological MS criteria in patients aged over 50 years were evaluated. The study found that MRI Barkhof criteria provided the best compromise for the diagnosis of MS patients with late onset. Other studies have reported different brain and spinal cord localisations in LOMS and TOMS patients, and more brain MRI inflammatory activity in TOMS than in LOMS patients $[17,18]$. A recently published study showed severe grey matter and brainstem atrophy in LOMS patients, with primary cognitive dysfunction [19]. The purpose of this study was to describe radiological characteristics based on conventional and non-conventional MRI techniques in LOMS patients.

\section{Patients and methods}

\section{Patients}

Twenty LOMS patients (15 women, 5 men) consecutively admitted to our neurological department were included in the study. Late onset MS patients were defined as patients who fulfilled McDonald's criteria of 2010 [21, 22], with first symptoms having appeared after their 50th birthday. Additionally, 17 (11 women, 6 men) typical age of onset MS (TOMS) patients, defined as patients who fulfilled the $2010 \mathrm{McD}$ onald criteria $[20,21]$ with first symptoms appearing between the ages of 20 and 45, were included in the study as a comparative group. All patients were assessed by an experienced neurologist and neurological status was measured by the Expanded Disability Status Scale (EDSS) [22]. The two groups were matched in terms of gender, disease duration, and EDSS score.

\section{Methods}

Each patient underwent magnetic resonance imaging on a 1.5 Tesla scanner (Avanto, Erlangen, Germany). In the first step, conventional MRI included dual-echo $(\mathrm{TR}=5,000 \mathrm{msec}, \mathrm{TE}=20 / 80 \mathrm{msec} ; 50$ slices, thickness $=3 \mathrm{~mm}$ gap $=0.0 \mathrm{~mm}$, matrix $154 \times 256$, and FOV $=250 \mathrm{~mm}$ ), T1-weighted Magnetization Prepared Rapid Gradient Echo (MPRAGE TR = $9.7 \mathrm{msec}, \mathrm{TE}=4 \mathrm{msec}$; eff thick $1.5 \mathrm{~mm}$, no partitions 164 , matrix $192 \times 256$ ), T1 weighted imaging with and without contrast administration $(\mathrm{TR}=30 \mathrm{~ms}$, $\mathrm{TE}=11 \mathrm{msec}$, thickness $=3 \mathrm{~mm}$ gap $=0.0 \mathrm{~mm}$, FOV $250 \mathrm{~mm}$, matrix $256 \times 256)$, fl 2D MT ON $(\mathrm{TR}=800 \mathrm{msec}, \mathrm{TE}=10 \mathrm{msec}$, thickness $=3 \mathrm{~mm}, \mathrm{FOV}=250 \mathrm{~mm}$, matrix $=159 \times 256) ; \mathrm{fl} 2 \mathrm{D}$ MT OFF $(\mathrm{TR}=800 \mathrm{msec}, \mathrm{TE}=10 \mathrm{msec}$, thickness $=3 \mathrm{~mm}$, FOV $=250 \mathrm{~mm}$, matrix $=159 \times 256)$ were acquired._In the second step, water suppressed proton magnetic resonance spectroscopy (H-MRS) was performed using a stimulated echo acquisition mode sequence STEAM (TE $=20 \mathrm{~ms}$, $\mathrm{TR}=6,000 \mathrm{~ms}, 64$ averages). Volume of interest (VOI) $=8 \mathrm{ml}$ was located in NAWM in left and right centrum semiovale far from white matter lesions. To avoid the inclusion of lesions, cerebrospinal fluid, or grey matter in VOI, the borders of the VOI at upper and lower slides were checked. Before positioning the voxel, global shimming of the whole brain was performed. After location, the VOI local magnetic field was homogenised by localised shimming on the water peak. Water suppression was achieved by means of chemically selective saturation pulse.

\section{MRI data analysis}

The volumes of focal lesions on T2-weighted images and T1-weighted images were measured with the application of the semi-automated technique (JavaImage, Xinapse version 5.0, UK) [21]. Brain atrophy was evaluated based on the MP-RAGE sequence. Brain Parenchyma Fraction (BPF), Grey Matter Fraction (GMF), and White Matter Fraction (WMF) were calculated using JavaImage software. Evaluation of normal appearing brain tissue (NABT) on the basis of magnetization transfer was conducted with the use of two gradient sequences: flash 2D without magnetization transfer and flash 2D with magnetisation transfer. With the use of adequate algorithms, a computer program calculated the magnetisation transfer ratio map (MTR) for particular analysed points [24]. Evaluation of the NABT was made with the use of MTR histograms. In the first stage, the brain tissue was extracted semiautomatically from cranial bones and the cerebrospinal fluid. Next, the MTR map was masked with focal lesions. After isolating them from the map, the remaining part of the image which showed only the NABT was used to make a MTR histogram. Mean value of MTR, peak position and peak height of the MTR histogram were analysed. 
Results of H-MRS were estimated using the linear combination model (LCModel, Provencher, 1993) [25]. Concentrations of the following metabolites were estimated using a basis set of 15 metabolites: creatine $(\mathrm{Cr})$, total $\mathrm{N}$-acetyl-aspartate compounds (tNAA $=\mathrm{N}$-acetyl-aspartate $(\mathrm{NAA})+\mathrm{N}$-acetyl-glutamate (NAAG)), choline-containing compounds (tCho) - including glycerophosphocholine and phosphocholine (GPC+PCh), and myoinositol (mIn). All subjects gave written, informed consent before entering the study. The study was approved by the Local Ethics Committee.

\section{Statistical analysis}

Statistical analysis was performed using Statistica 10th CSS. The results of the quantitative variables are presented as a mean \pm SD (standard deviation), and median \pm SD, as required.

The data was verified for normality (Shapiro-Wilk test) of distribution and equality of variances. To compare the means, the Student's t-test was used when the distribution was normal and in other cases U Mann-Whitney test was used to compare received average values. To exclude the impact of age on the brain atrophy measures (BPF, GMF, WMF) ANCOVA analysis with age as a covariate was conducted. However, for all three parameters (BPF, WMF, GMF) assumptions of ANCOVA was not met (linearity of relationship between outcome variables i.e. brain atrophy parameters and covariate i.e. age as well as lack of normal distribution for outcome variables and presence of outliers).

Therefore, we could not conduct ANCOVA analysis and we have checked the presence of relationship (linear regression) and correlation (Spearman's correlation coefficient) between age and each of the brain atrophy parameters for both groups (TOMS and LOMS). The limit of statistical significance was set at $\mathrm{p}<0.05$ for all the analyses.

\section{Results}

\section{Demographic characteristics of the LOMS and TOMS patients}

The mean age of LOMS patients was 57.8 years ( $\mathrm{SD} \pm 4.7$ ) and the mean age of TOMS patients was 34.3 years $(\mathrm{SD} \pm 6.7)$. None of the patients had comorbidities such as hypertension, hyperlipidemia or heart disease. None of the patients were undergoing immunomodulatory or immunosuppression treatment.

The demographic and clinical characteristics of the MS patients are set out in Table 1.

\section{MRI analysis}

\section{Conventional MRI results}

The T2 and T1-lesion volume was similar in both groups ( $\mathrm{p}=0.3, \mathrm{p}=0.1$ respectively). T1 $/ \mathrm{T} 2$ volume ratio was higher in the LOMS group but not statistically significantly (Tab. 2).

\section{Nonconventional MRI results}

Analysis of the BPF in the LOMS and TOMS groups showed that in LOMS BPF was significantly lower than in TOMS $(\mathrm{p}<0.01)$. Similarly, the LOMS group was characterised by lower GMF values than the TOMS group $(\mathrm{p}=0.008)$. There was no statistical difference between the groups in WMF $(\mathrm{p}=0.527)$ (Tab. 2). There was no correlation between age and any of the three brain atrophy parameters either in the TOMS, nor in the LOMS group (LOMS: BPF $r=0.13, p=0.598$; GMF $r=-0.25$, $\mathrm{p}=0.287 ; \mathrm{WMFr}=0.32, \mathrm{p}=0.163$, TOMS: $\mathrm{BPFr}=-0.06, \mathrm{p}=0.815$, GMF $\mathrm{r}=-0,14 . \mathrm{p}=0.585$; WMF $\mathrm{r}=0.07, \mathrm{p}=0.775$ respectively).

Analysis of MTR histogram data revealed that in the LOMS group mean MTR value was significantly lower than in the TOMS group ( $\mathrm{p} \leq 0.001$ ). We also found that the peak height and peak position were significantly lower in LOMS patients compared to TOMS patients ( $\mathrm{p}=0.018, \mathrm{p}<0.001$ respectively) (Tab. 2). Analysis of H-MRS parameters between the LOMS and TOMS groups showed that in LOMS patients concentrations of NAA+NAAG was significantly lower than in TOMS patients $(\mathrm{p}=0.009)$. Concentrations of the remaining H-MRS metabolites from the NAWM were comparable in both groups $(\mathrm{p}>0.05)$ (Tab. 2).

\section{Discussion}

In this study, we assessed differences in MRI characteristics between late and normal age of onset multiple sclerosis patients. We found that LOMS patients differ from TOMS patients in non-conventional MRI characteristics.

Table 1. Demographic and clinical characteristics of LOMS and TOMS groups. Table presents mean values; brackets contain standard deviation values and size of group $(n)$

\begin{tabular}{llll}
\multicolumn{1}{c}{ Variables } & \multicolumn{1}{c}{ LOMS $\mathbf{n = 2 0}$} & \multicolumn{1}{c}{ TOMS $\mathbf{n = 1 7}$} & \multicolumn{1}{c}{ : } \\
\hline Sex M/F & $5 / 15(25 \%)$ & $6 / 11(35 \%)$ & $<001$ \\
Age at occurrence of first symptoms (years) & $53.7(3.6)$ & $29.8(7.7)$ & $<0.001$ \\
Duration of MS (years) & $5.0(3.15)$ & $5.12(3.77)$ & 0.92 \\
EDSS (median) & $3.5(1.56)$ & $3.5(1.53)$ & 0.89
\end{tabular}

LOMS — Late Onset Multiple Sclerosis; TOMS — Typical Onset Multiple Sclerosis; EDSS — Expanded Disability Status Scale 
Table 2. Conventional and non-conventional MRI results in LOMS and TOMS groups

\begin{tabular}{|c|c|c|c|c|}
\hline Variables & & LOMS n = 20 & TOMS $n=17$ & $\mathbf{P}$ \\
\hline $\begin{array}{l}\text { Volume of lesions on T2-weighted } \\
\text { images }\left[\mathrm{mm}^{3}\right] \pm S D\end{array}$ & & $11,197.2 \pm 10,018.3$ & $10,157.5 \pm 12,489.8$ & 0.3 \\
\hline $\begin{array}{l}\text { Volume of lesions on T1-weighted } \\
\text { images }\left[\mathrm{mm}^{3}\right] \pm S D\end{array}$ & & $5,357.7 \pm 5,213.1$ & $4,044.34 \pm 7,094.6$ & 0.1 \\
\hline $\mathrm{T} 1$ volume/T2 volume ratio $\pm \mathrm{SD}$ & & $0.72 \pm 1.23$ & $0.41 \pm 0.32$ & 0.88 \\
\hline \multicolumn{5}{|l|}{ Brain Fractions } \\
\hline & BPF & $0.752 \pm 0.03$ & $0.82 \pm 0.07$ & $<0.001$ \\
\hline & GMF & $0.4 \pm 0.03$ & $0.43 \pm 0.13$ & 0.008 \\
\hline & WMF & $0.36 \pm 0.05$ & $0.37 \pm 0.16$ & 0.572 \\
\hline \multicolumn{5}{|l|}{ MTR Histogram } \\
\hline & Peak location & $34.94 \pm 1.25$ & $42.19 \pm 1.94$ & $<0.001$ \\
\hline & Peak height & $56.79 \pm 6.13$ & $67.2 \pm 12.19$ & 0.018 \\
\hline & MTR mean value & $28.82 \pm 1.58$ & $36.72 \pm 2.71$ & $<0.001$ \\
\hline \multicolumn{5}{|l|}{ H-MRS } \\
\hline & mean NAA+NAAG & $7.1 \pm 1.56$ & $8.81 \pm 1.42$ & 0.009 \\
\hline & mean In & $4.88 \pm 1.40$ & $4.49 \pm 1.13$ & 0.346 \\
\hline & mean GPC+PCh & $1.59 \pm 0.41$ & $1.65 \pm 0.31$ & 0.563 \\
\hline
\end{tabular}

LOMS - Late Onset Multiple Sclerosis; TOMS — Typical Onset Multiple Sclerosis; SD — standard deviation; BPF — Brain Parenchymal Fraction; GMF — Grey Matter Fraction; WMF — White Matter Fraction; MTR - Magnetic Transfer Ratio; H-MRS - Water Suppressed Proton Magnetic Resonance Spectroscopy; $\mathrm{NAA+NAAG}-\mathrm{N}$-acetyl-aspartate (NAA) + N-acetyl-glutamate (NAAG); GPC+PCh - glycerophosphocholine and phosphocholine (GPC+PCh); In - myoinositol (mln)

To the best of our knowledge, there are no published results concerning a multiparametric MRI comparison of a LOMS and TOMS study. Most of the published results concerning differences between LOMS and TOMS patients have referred to the clinical presentation $[5-8,11-13]$ However, it seems that an MRI study could add more data that would explain the observed differences.

We did not find a difference of T1 and T2-lesion volume between the LOMS and TOMS groups. However, T1/T2 lesion volume ratio was higher in the LOMS than in the TOMS group. Results of published studies indicate that around one third of the lesions visible on T2-weighted images correspond to hypointensities ('black holes') on T1-weighted sequences [26]. Additionally, a comparative analysis of the histopathology and MRI examination revealed that focal hypointense lesions on T1-weighted images are associated with a decrease in axonal density and axonal loss [27]. Higher T1/T2 lesion volume ratio in LOMS might indicate that there is a greater contribution of axonopathy on pathology in these types of MS. We speculate that the process which takes place in focal changes in LOMS is more destructive than in TOMS patients, with more dominant axonal damage.

Another interesting finding comes from our brain atrophy analysis. Brain Parenchyma Fraction was significantly lower in our LOMS group compared to our TOMS group. The findings of a longitudinal MRI analysis throughout the lifespan in healthy subjects revealed that brain volume change is an ongoing process [28]. Brain volume increases in childhood and adolescence until the age of 13 years, while between 18 and 35 years of age there is a second period of brain volume growth, or at least stability. After the age of 35, there is a steady brain volume loss $(0.2 \%$ per year) accelerating to $0.5 \%$ per year at age 60 [28].

Additionally, recently published results showed that percentage of brain volume change was also associated with magnetic field strength [29]. In multiple sclerosis patients, the rate of brain atrophy is faster than in healthy age-matched controls $(0.5 \% /-1.35 \%$ /year vs $0.1-0.3 \%$ /year) respectively [30]. Brain volume loss in MS patients depends on different factors such as disease stage, neurological disability, type of pharmacological treatment, and other factors unrelated to the disease [31]. Although the LOMS group was significantly older than the TOMS group, we exclude the impact of age as a covariant on differences of brain atrophy parameters between the LOMS and TOMS groups.

A lack of correlation between age and brain atrophy parameters confirms that the significant difference in brain atrophy parameters between TOMS and LOMS is not related to differences in age between the groups. All participants were scanned on the same scanner $(1.5 \mathrm{~T})$ with no up-grade during the study period and with the same MRI protocol. Therefore, we can exclude the impact of technical factors on brain volume differences between LOMS and TOMS patients. 
Because cardiovascular risk factors such as hypertension, hyperlipidemia and heart disease are associated with an increased number of white matter focal abnormalities and decreased whole-brain and grey matter volume, we excluded from our final analysis MS patients with these comorbidities [32-34].

Finally, our groups were also adequately matched in terms of gender, disease duration and disability level (EDSS). Because we can exclude the impact of these factors of the final results on our findings, we can speculate that the pathological processes contributing to the pathology of LOMS and TOMS are different. Lower BPF in LOMS patients indicates that in this type of MS the neurodegenerative process is much more advanced than in TOMS. We can also assume that the process of brain plasticity is less effective in LOMS than in TOMS.

Another interesting result came from tissue brain fraction analysis. Grey matter fraction in LOMS patients was significantly lower than in the TOMS group. We did not find such differences when comparing white matter fraction. This may indicate that in the LOMS group grey matter atrophy is much more advanced than in TOMS patients.

Based on published results [35-37], we can also speculate that in the LOMS group grey matter atrophy precedes white matter damage. These findings, and the lack of WMF atrophy, may also suggest that in LOMS patients a neurodegenerative process in grey matter makes a much greater contribution to MS pathology and global brain atrophy than in TOMS patients. Grey matter atrophy in our LOMS group is in line with previously published results which showed that in LOMS patients with cognitive dysfunction grey matter damage is a very characteristic MRI finding [19]

The results of the magnetization transfer imaging support another argument for differences between LOMS and TOMS patients. In the LOMS group, the peak location, the peak height, and the mean MTR value were all lower than in the TOMS group. Lower MTR values signify greater diminution of structure integrity and higher intensity of pathological processes [38]. Especially in MS, it shows not only demyelination but also axonal loss [39]. Because patients were adequately matched with regard to disease duration and neurological deficits, we assume that damage of NAWM in LOMS patients is much more advanced than in TOMS patients. We can also speculate that damage of NAWM has a greater impact on MS pathology in LOMS than in TOMS. We could also speculate that subclinical NAWM changes may occur earlier in LOMS than in TOMS. This might also imply a different type of mechanism responsible for the pathology in the central nervous system in those two types of MS.

Our results from H-MRS spectroscopy added more information concerning LOMS and TOMS differences. We found that the tNAA concentration was significantly lower in the LOMS group than in the TOMS group. Based on previously published results which detected that decreased tNAA concentration correlates with axonal damage and loss, lower tNAA in the LOMS group with normal concentration of other metabolites in our study seems to be further evidence of more widespread axonal pathology in LOMS than in TOMS patients.

The very interesting question arises as to why the first symptoms in LOMS occur later than in TOMS. It seems possible that LOMS patients, earlier than TOMS patients, are affected by pathological processes but on a subclinical level. The dynamic of this process seems to be slower in LOMS than in TOMS. We can also assume that natural brain damage that occurs in older patients may contribute to the first presentation of MS. We can also speculate that for a long period, the reparatory processes are more effective in LOMS than in TOMS patients, which prevents an earlier occurrence of neurological symptoms. Along with the course of the disease and with advancing age, the mechanism of remyelination declines, prompting the presentation of MS clinical symptoms [3].

Our study has some limitations. It is a description of a small population of LOMS and TOMS patients; due to this fact, the presence of multiple sclerosis phenotypes (RRMS vs. PPMS) in both groups of patients was not included. We are aware that some differences in MRI of these two forms of MS may affect the obtained results in conventional MRI techniques. Nonetheless, to the best of our knowledge, this is the first description of conventional and non-conventional MRI in a LOMS population.

\section{Conclusions}

Differences in MRI presentation between LOMS and TOMS patients confirm the heterogenic character of MS, with probably more advanced axonal pathology in LOMS than in TOMS patients. Our results may contribute to a better understanding of the differences in the pathogenesis of various types of MS, and we hope may support improved therapeutic decision-making.

\section{Conflict of interests: None}

\section{Acknowledgment}

We would like to thank Professor Krzysztof Selmaj for his scientific and methodological contribution to this project. Profesor Selmaj was also the supervisor of Dr Jasek's PhD thesis. The results presented in this article come from Dr Jasek's $\mathrm{PhD}$ research project. Research was funded from Doctorship grant N N402 193435, Contract number: 1934/B/P01/2008/35.

\section{References}

1. Olek MJ, Dawson DM. Multiple sclerosis and other inflammatory demyelinating diseases of the central nervous system. W: Bradley W.G., Daroff F.B. and Fenichel G.M., ed. : Neurology in Clinical Practice. ; 1999.

2. Kremenchutzky M, Cottrell D, Rice G, et al. The natural history of multiple sclerosis: a geographically based study. 7. Progressive-relapsing and relapsing-progressive multiple sclerosis: a re-evaluation. Brain. 1999; 122 ( Pt 10): 1941-1950, doi: 10.1093/brain/122.10.1941, indexed in Pubmed: 10506095. 
3. Confavreux C, Vukusic S, Moreau T, et al. Relapses and progression of disability in multiple sclerosis. N Engl J Med. 2000; 343(20): 1430-1438, doi: 10.1056/NEJM200011163432001, indexed in Pubmed: 11078767.

4. Tremlett $\mathrm{H}$, Devonshire $\mathrm{V}$. Is late-onset multiple sclerosis associated with a worse outcome? Neurology. 2006; 67(6): 954-959, doi: 10.1212/01.wnl.0000237475.01655.9d, indexed in Pubmed: 17000960.

5. Kis B, Rumberg B, Berlit P. Clinical characteristics of patients with late-onset multiple sclerosis. J Neurol. 2008; 255(5): 697-702, doi: 10.1007/s00415-008-0778-x, indexed in Pubmed: 18283394.

6. Polliack ML, Barak Y, Achiron A. Late-onset multiple sclerosis. J Am Geriatr Soc. 2001; 49(2): 168-171, doi: 10.1046/j.15325415.2001.49038.x, indexed in Pubmed: 11207871.

7. Martinelli $\mathrm{V}$, Rodegher M, Moiola L, et al. Late onset multiple sclerosis: clinical characteristics, prognostic factors and differential diagnosis. Neurol Sci. 2004; 25 Suppl 4: S350-S355, doi: 10.1007/s10072004-0339-8, indexed in Pubmed: 15727232.

8. de Seze J, Delalande S, Michelin E, et al. [Late onset multiple sclerosis]. Rev Neurol (Paris). 2002; 158(11): 1082-1087, indexed in Pubmed: 12451340.

9. Koch-Henriksen N, Thygesen LC, Stenager E, et al. Incidence of MS has increased markedly over six decades in Denmark particularly with late onset and in women. Neurology. 2018; 90(22): e1954-e1963, doi: 10.1212/WNL.0000000000005612, indexed in Pubmed: 29720546.

10. Lublin FD, Reingold SC, Cohen JA, et al. Defining the clinical course of multiple sclerosis: the 2013 revisions. Neurology. 2014; 83(3): 278286, doi: 10.1212/WNL.0000000000000560, indexed in Pubmed: 24871874.

11. Tremlett H, Paty D, Devonshire V. The natural history of primary progressive MS in British Columbia, Canada. Neurology. 2005; 65(12): 1919-1923, doi: 10.1212/01.wnl.0000188880.17038.1d, indexed in Pubmed: 16380613.

12. Arias M, Dapena D, Arias-Rivas S, et al. Late onset multiple sclerosis. Neurologia. 2011; 26(5): 291-296, doi: 10.1016/j.nrl.2010.09.008, indexed in Pubmed: 21163234.

13. Shirani A, Zhao Y, Petkau J, et al. Multiple sclerosis in older adults: the clinical profile and impact of interferon Beta treatment. Biomed Res Int. 2015; 2015: 451912, doi: 10.1155/2015/451912, indexed in Pubmed: 25922836.

14. Guillemin F, Baumann C, Epstein J, et al. LORSEP Group. Older Age at Multiple Sclerosis Onset Is an Independent Factor of Poor Prognosis: A Population-Based Cohort Study. Neuroepidemiology. 2017; 48(3-4): 179-187, doi: 10.1159/000479516, indexed in Pubmed: 28793296.

15. Lotti $C B$, Oliveira AS, Bichuetti $D B$, et al. Late onset multiple sclerosis: concerns in aging patients. Arq Neuropsiquiatr. 2017; 75(7): 451-456, doi: 10.1590/0004-282X20170070, indexed in Pubmed: 28746432.

16. Alroughani R, Akhtar S, Ahmed S, et al. Is Time to Reach EDSS 6.0 Faster in Patients with Late-Onset versus Young-Onset Multiple Sclerosis? PLoS One. 2016; 11(11): e0165846, doi: 10.1371/journal. pone.0165846, indexed in Pubmed: 27802328.

17. Roohani P, Emiru T, Carpenter A, et al. Late onset multiple sclerosis: Is it really late onset? Mult Scler Relat Disord. 2014; 3(4): 444-449, doi: 10.1016/j.msard.2014.02.004, indexed in Pubmed: 25877055.

18. D'Amico E, Patti F, Zanghì A, et al. Late-onset and young-onset relapsing-remitting multiple sclerosis: evidence from a retrospective longterm follow-up study. Eur J Neurol. 2018; 25(12): 1425-1431, doi: 10.1111/ene.13745, indexed in Pubmed: 29956427.
19. Calabrese M, Reynolds R, Magliozzi R, et al. Regional Distribution and Evolution of Gray Matter Damage in Different Populations of Multiple Sclerosis Patients. PLoS One. 2015; 10(8): e0135428, doi: 10.1371/ journal.pone.0135428, indexed in Pubmed: 26267665.

20. McDonald WI, Compston A, Edan G, et al. Recommended diagnostic criteria for multiple sclerosis: guidelines from the International Panel on the diagnosis of multiple sclerosis. Ann Neurol. 2001; 50(1): 121-127, doi: 10.1002/ana.1032, indexed in Pubmed: 11456302.

21. Polman $\mathrm{CH}$, Reingold SC, Banwell B, et al. Diagnostic criteria for multiple sclerosis: 2010 revisions to the McDonald criteria. Ann Neurol. 2011; 69(2): 292-302, doi: 10.1002/ana.22366, indexed in Pubmed: 21387374.

22. Kurtzke JF. Rating neurologic impairment in multiple sclerosis: an expanded disability status scale (EDSS). Neurology. 1983; 33(11): 1444-1452, doi: 10.1212/wnl.33.11.1444, indexed in Pubmed: 6685237.

23. Java Image, Xinapse. : UK.

24. van Buchem MA, Udupa JK, McGowan JC, et al. Global volumetric estimation of disease burden in multiple sclerosis based on magnetization transfer imaging. AJNR Am J Neuroradiol. 1997; 18(7): 1287-1290, indexed in Pubmed: 9282856.

25. Provencher SW. Estimation of metabolite concentrations from localized in vivo proton NMR spectra. Magn Reson Med. 1993; 30(6): 672-679, doi: 10.1002/mrm.1910300604, indexed in Pubmed: 8139448.

26. Turano G, Jones SJ, Miller DH, et al. Correlation of SEP abnormalities with brain and cervical cord MRI in multiple sclerosis. Brain. 1991; 114 ( Pt 1B): 663-681, doi: 10.1093/brain/114.1.663, indexed in Pubmed: 2004262.

27. Lycklama à Nijeholt G, Barkhof F. Differences between subgroups of MS: MRI findings and correlation with histopathology. J Neurol Sci. 2003; 206(2): 173-174, doi: 10.1016/s0022-510x(02)00336-2, indexed in Pubmed: 12559506.

28. Hedman AM, van Haren NEM, Schnack HG, et al. Human brain changes across the life span: a review of 56 longitudinal magnetic resonance imaging studies. Hum Brain Mapp. 2012; 33(8): 1987-2002, doi: 10.1002/hbm.21334, indexed in Pubmed: 21915942.

29. Battaglini M, Gentile G, Luchetti L, Giorgio A, Vrenken H, Barkhof F, Cover KS, Bakshi R, Chu R, Sormani MP, Enzinger C, Ropele S, Ciccarelli 0 , Wheeler-Kingshott C, Yiannakas M, Filippi M, Rocca MA, Preziosa P, Gallo A, Bisecco A, Palace J, Kong Y, Horakova D, Vaneckova M, Gasperini C, Ruggieri S, De Stefano N. MAGNIMS Study Group.

30. De Stefano N, Airas L, Grigoriadis N, et al. Clinical relevance of brain volume measures in multiple sclerosis. CNS Drugs. 2014; 28(2): 147-156, doi: 10.1007/s40263-014-0140-z, indexed in Pubmed: 24446248.

31. Giorgio A, Battaglini M, Smith SM, et al. Brain atrophy assessment in multiple sclerosis: importance and limitations. Neuroimaging Clin N Am. 2008; 18(4): 675-86, xi, doi: 10.1016/j.nic.2008.06.007, indexed in Pubmed: 19068408.

32. Kappus N, Weinstock-Guttman B, Hagemeier J, et al. Cardiovascular risk factors are associated with increased lesion burden and brain atrophy in multiple sclerosis. J Neurol Neurosurg Psychiatry. 2016; 87(2): 181-187, doi: 10.1136/jnnp-2014-310051, indexed in Pubmed: 25722366.

33. Horakova D, Zivadinov R, Weinstock-Guttman B, et al. Environmental factors associated with disease progression after the first demyelinating event: results from the multi-center SET study. PLoS One. 2013; 8(1): e53996, doi: 10.1371/journal.pone.0053996, indexed in Pubmed: 23320113. 
34. Weinstock-Guttman B, Zivadinov R, Horakova D, et al. Lipid profiles are associated with lesion formation over 24 months in interferon- $\beta$ treated patients following the first demyelinating event. J Neurol Neurosurg Psychiatry. 2013; 84(11): 1186-1191, doi: 10.1136/jnnp2012-304740, indexed in Pubmed: 23595944.

35. Tur C, Penny S, Khaleeli Z, et al. Grey matter damage and overall cognitive impairment in primary progressive multiple sclerosis. Mult Scler. 2011; 17(11): 1324-1332, doi: 10.1177/1352458511410341, indexed in Pubmed: 21803874.

36. Roosendaal SD, Bendfeldt K, Vrenken H, et al. Grey matter volume in a large cohort of MS patients: relation to MRI parameters and disability. Mult Scler. 2011; 17(9): 1098-1106, doi: 10.1177/1352458511404916, indexed in Pubmed: 21586487.
37. Furby J, Hayton T, Altmann D, et al. A longitudinal study of MRIdetected atrophy in secondary progressive multiple sclerosis. J Neurol. 2010; 257(9): 1508-1516, doi: 10.1007/s00415-010-5563-y, indexed in Pubmed: 20437181.

38. Filippi M, Rocca MA, Minicucci L, et al. Magnetization transfer imaging of patients with definite MS and negative conventional MRI. Neurology. 1999; 52(4): 845-848, doi: 10.1212/wnl.52.4.845, indexed in Pubmed: 10078737.

39. van Waesberghe JH, Kamphorst W, De Groot CJ, et al. Axonal loss in multiple sclerosis lesions: magnetic resonance imaging insights into substrates of disability. Ann Neurol. 1999; 46(5): 747-754, doi: 10.1002/1531-8249(199911)46:5<747::aid-ana10>3.3.co;2-w, indexed in Pubmed: 10553992. 